\title{
Sondas, cateteres e outros aparatos médicos na radiografia de tórax
}

\author{
Tubes, catheters and other medical devices on chest radiography

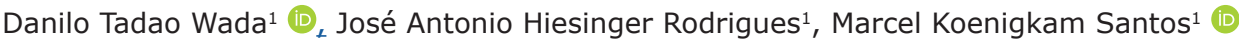

\begin{abstract}
RESUMO
O exame de radiografia simples $(\mathrm{RX})$ do tórax é utilizado para verificação do posicionamento adequado de vários tipos de aparatos médicos, além de auxiliar na detecção de complicações relacionadas à sua implantação e uso. Principalmente nos pacientes criticamente doentes, com suporte cardiovascular ou ventilação mecânica, a realização do RX é importante na avaliação de tubos endotraqueais, sondas de alimentação, cateteres vasculares e drenos de tórax. O posicionamento inadequado desses dispositivos pode levar a complicações sérias que muitas vezes não são clinicamente evidentes. Outros aparatos médicos, mesmo que na parede torácica ou externos ao corpo do paciente, podem ser identificados no exame de RX, sendo importante saber reconhece-los para não confundir com imagens de corpo estranho ou outro tipo de lesão torácica. Neste texto, serão abordados os aspectos radiográficos das principais sondas, tubos e cateteres, vasculares e não vasculares, discutindo o posicionamento correto e algumas complicações identificáveis ao exame de RX de tórax.
\end{abstract}

Palavras-chave: Radiografia Simples. Tórax. Cateteres. Dispositivos Médicos.

\begin{abstract}
Conventional chest radiography is routinely used to check the position of many medical devices, also allowing us to identify a bunch of complications related to them. Mostly in the critically ill patients with cardiovascular support or mechanical ventilation, chest radiography is important in the evaluation of endotracheal tubes, alimentary probes, vascular catheters and thoracic tubes. The correct interpretation of findings related to these devices is essential, as many of the complications can be clinically missed.Other medical devices superposed to the chest wall should be correctly identified for avoiding misinterpretations leading to incorrect diagnoses like intrathoracic strange bodies or other lesions. In this article, it will be presented some of the imaging aspects of the main probes, catheters and devices (vascular and non-vascular), discussing the correct positioning and some of the complications that can be found in chest radiography.
\end{abstract}

Keywords: Conventional Radiography. Chest. Catheters. Medical Devices.

1. Médico assistente da disciplina de Radiologia Torácica e Cardiovascular. Centro de Ciências das Imagens e Física Médica, Hospital das Clínicas da Faculdade de Medicina de Ribeirão Preto da Universidade de São Paulo, Ribeirão Preto (SP), Brasil.

2. Docente responsável pela disciplina de Radiologia Torácica e Cardiovascular. Fundação de Apoio ao Ensino, Pesquisa e Assistência do Hospital das Clínicas da Faculdade de Medicina de Ribeirão Preto da Universidade de São Paulo (FAEPA), Ribeirão Preto (SP), Brasil.

M Danilo Tadao Wada. Avenida Bandeirantes, 3900 - Campus Universitário. CEP: 14049-900. Ribeirão Preto SP, Brasil. dwada@hcrp.usp.br | Recebido em: 17/02/2019 | Aprovado em: 26/04/2019 


\section{INTRODUÇÃO}

$\mathrm{O}$ exame de radiografia simples ( $R X)$ do tórax é utilizado para verificação do posicionamento adequado de vários tipos de aparatos médicos, de monitorização ou suporte, vasculares e extra vasculares $^{1}$. Dentre eles, destacamos os tubos endotraqueais, sondas digestivas, drenos pleurais ${ }^{2,3}$, cateteres venosos e arteriais e os marcapassos cardíacos ${ }^{4,5}$.

Além de verificar o posicionamento adequado do dispositivo médico, o RX auxilia na detecção de complicações relacionadas à passagem ou instalação do mesmo e complicações tardias relacionadas à sua presença ${ }^{3}$.

Nos pacientes criticamente doentes, com suporte cardiovascular ou ventilação mecânica, o colégio americano de radiologia recomenda a realização diária do $\mathrm{RX}$ de tórax ${ }^{1}$, além da realização imediata do exame para todos os pacientes que foram submetidos a colocação de tubos endotraqueais, sondas de alimentação, cateteres vasculares e drenos de tórax. Estas recomendações são feitas porque o mal posicionamento desses dispositivos pode levar a sérias complicações, que muitas vezes não são clinicamente evidentes.

Desta maneira, é importante conhecer não somente 0 aspecto radiográfico habitual destes aparatos, como saber identificar possíveis complicações relacionadas ao seu uso ${ }^{2,4}$.

\section{OBJETIVOS GERAIS}

Após a leitura deste texto, o leitor deverá ser capaz de:
- reconhecer o aspecto radiográfico dos principais aparatos médicos utilizados no tórax, como tubos, sondas e cateteres

- $\quad$ saber identificar o posicionamento adequado destes dispositivos

- conhecer as principais complicações relacionados à sua instalação e permanência

\section{Aparatos não vasculares}

O exame de RX de tórax deve ser realizado para verificar a posição e sinais de complicações dos seguintes aparatos não vasculares:

- tubo endotraqueal

- tubo de traqueostomia

- $\quad$ sonda gástrica

- $\quad$ sonda entérica

- dreno de tórax

\section{Tubo endotraqueal e traqueostomia}

A intubação traqueal é realizada para manter a via aérea pérvia e auxiliar na ventilação de pacientes com hipoventilação ou hipoxemia. O posicionamento correto dos tubos endotraqueais deve ser verificado logo após sua introdução. No RX de tórax, a extremidade distal do tubo deve estar no terço médio da traqueia torácica, distando aproximadamente $5 \mathrm{~cm}$ da carina ${ }^{2,3}$ (não menos que 3 , não mais que $7 \mathrm{~cm}$ ). $O$ exame deve ser realizado com a cabeça do paciente em posição neutra, pois a flexão ou extensão pode mudar em até $2 \mathrm{~cm}$ a posição do tubo (figura 1). 


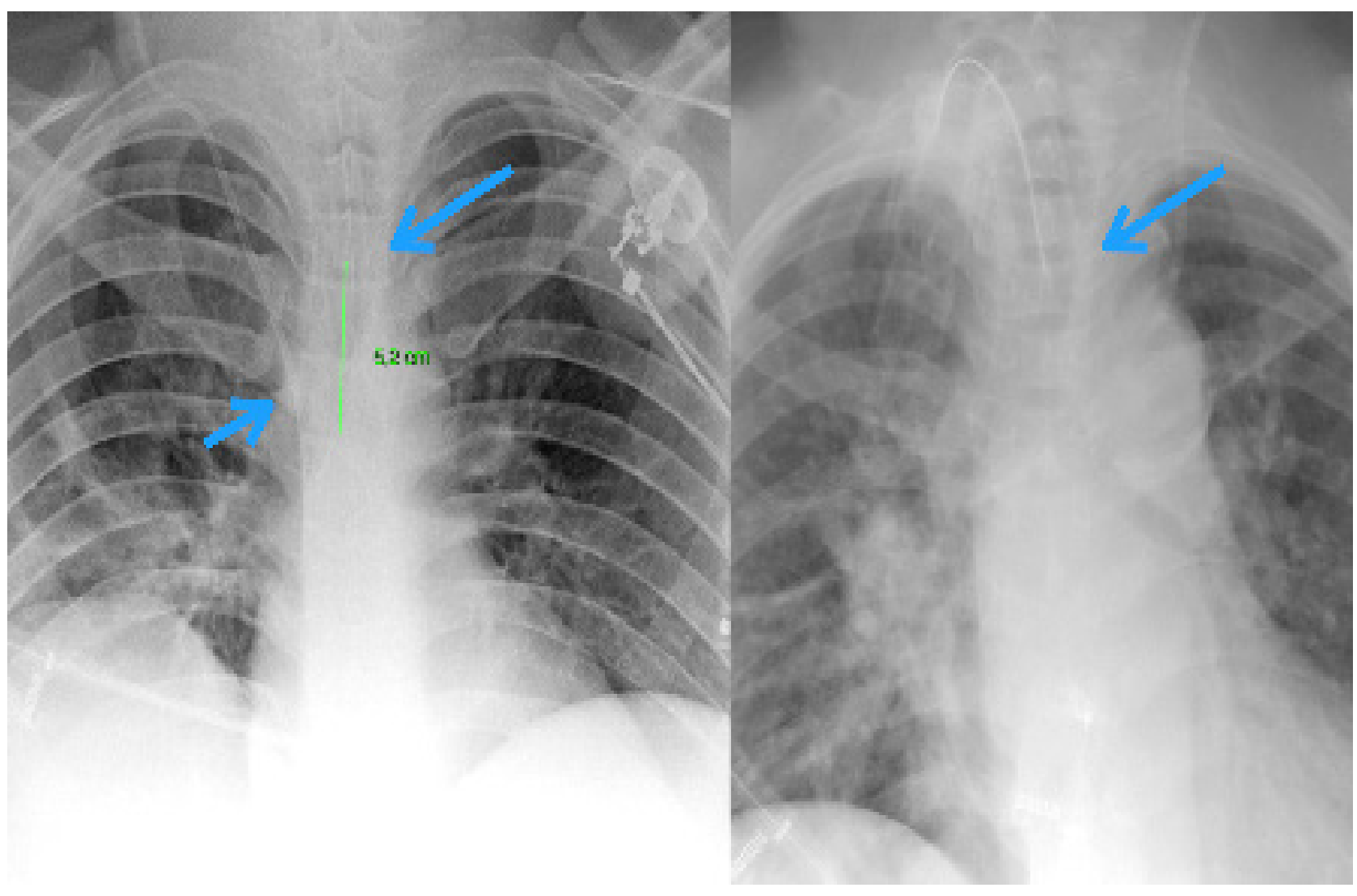

Figura 1: A esquerda, tubo endotraqueal adequadamente posicionado, com extremidade distal a 5,2 cm da carina. Notar também o cateter de acesso venoso central via jugular interna direita com extremidade em projeção da veia cava superior e fios de monitorização cardíaca. A direita, cânula de traqueostomia adequadamente posicionada.

A traqueostomia é realizada diante da necessidade de intubação prolongada. A ponta do tubo (ou cânula) de traqueostomia deve estar localizada aproximadamente na metade da distância entre a estomia e a carina ${ }^{6,7}$ (figura 1). Diferentemente da avaliação do tubo orotraqueal, a avaliação radiográfica da traqueostomia independe da posição da cabeça do paciente. Pequena quantidade de enfisema subcutâneo ou mesmo pequeno pneumomediastino pode ser identificado logo após a traqueostomia e passagem da cânula, sem repercussão clínica².

A extremidade do tubo endotraqueal deve estar a aproximadamente $5 \mathrm{~cm}$ da carina. Já o tubo de traqueostomia deve estar "no meio do caminho" entre a estomia e a carina.

Se o tubo endotraqueal estiver muito alto, há o risco de extubação, ventilação ineficiente, distensão gástrica e lesão das cordas vocais. Se o tubo estiver muito baixo, pode ocorrer intubação seletiva, principalmente para o brônquio fonte direito, que é mais verticalizado, levando a atelectasia pulmonar parcial ou completa2,8. A intubação seletiva também aumenta o risco de pneumotórax contralateral, devido a hiperinsuflação compensatória (figura 2). Uma complicação importante é a laceração da traqueia, principalmente da porção membranosa posterior, levando a pneumomediastino e pneumotórax ${ }^{9}$. Neste caso, a tomografia computadorizada (TC) é importante para demonstrar a localização e extensão da lesão traqueal $^{2}$ (figura 3 ). Outra possível complicação é o posicionamento do tubo no esôfago, podendo inclusive levar a perfuração esofágica, com pneumomediastino e posterior mediastinite ${ }^{2}$. Quando o tubo está no esôfago, o RX mostra o tubo lateralmente à coluna aérea normal da traqueia, ou se estendendo inferiormente à carina, muito comumente com distensão aérea do estômago ${ }^{8,10}$ 


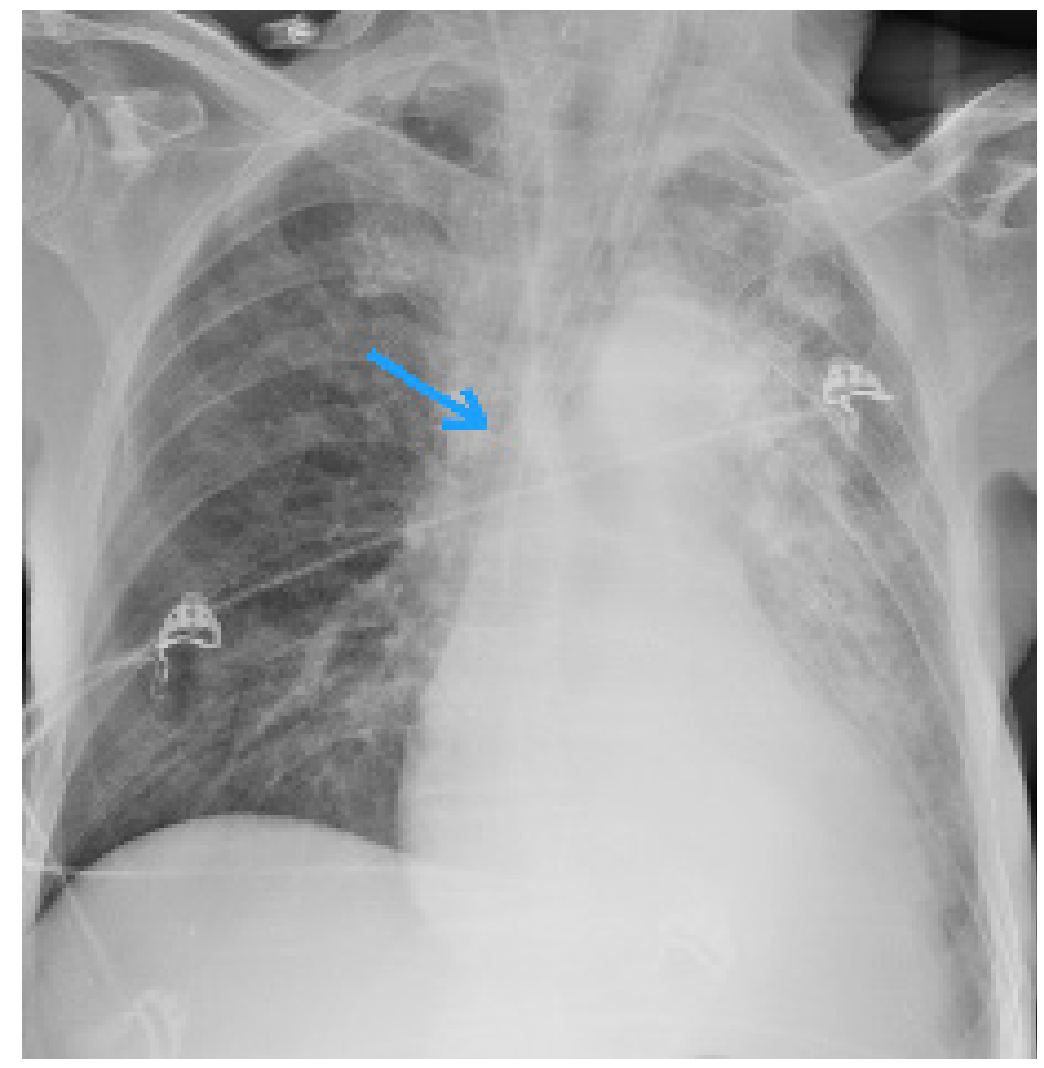

Figura 2: Intubação seletiva em brônquio fonte direito, com atelectasia parcial do pulmão esquerdo e desvio mediastinal para a esquerda. Há também cateter venoso central bem posicionado e fios de monitorização cardíaca.

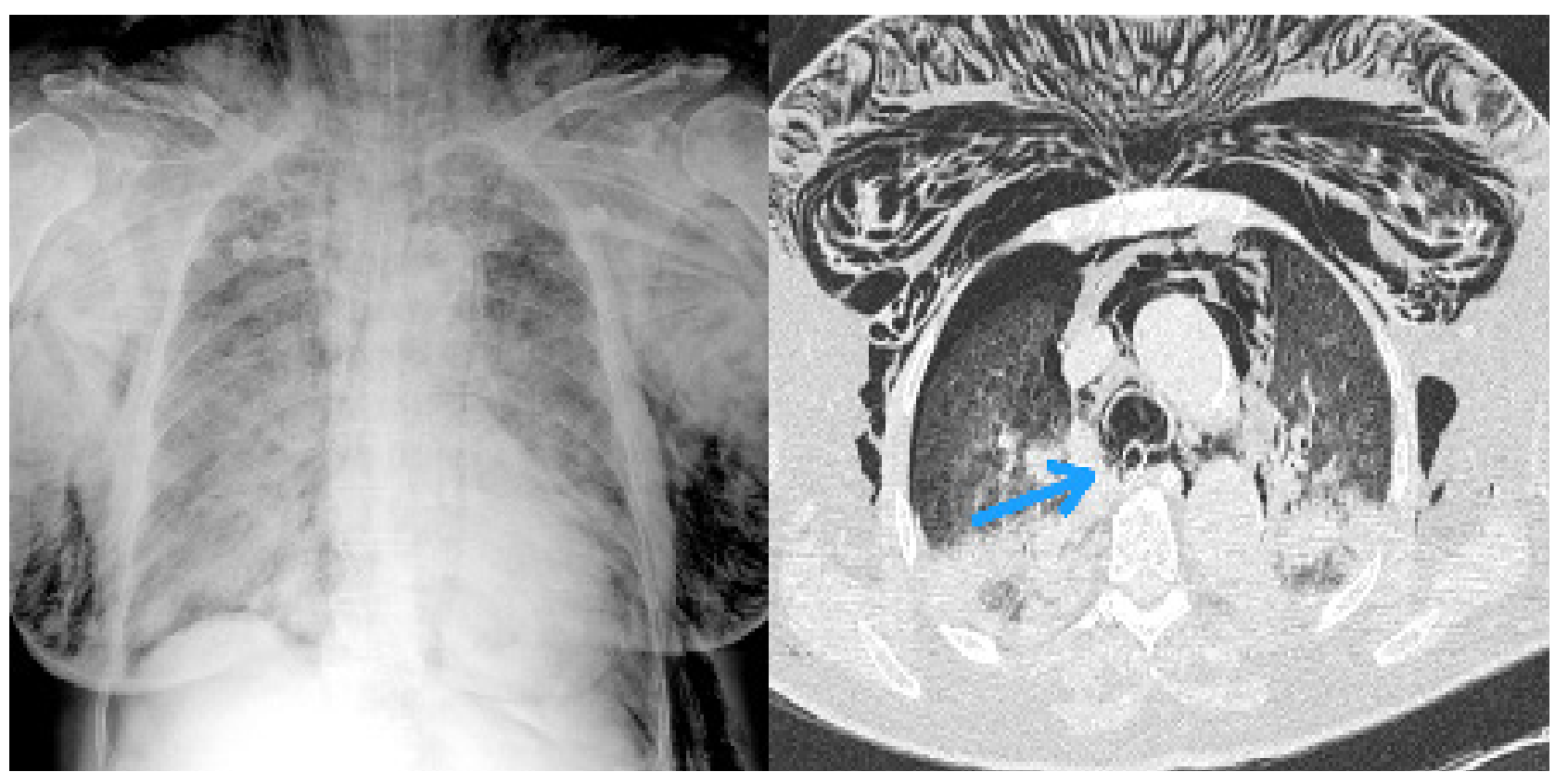

Figura 3: Controle radiográfico pós intubação traqueal mostra extenso enfisema de partes moles, pneumotórax a direita e pneumomediastino (desenhando a aorta descendente e coração). A direita, a TC mostra a laceração da membrana posterior da traqueia pelo tubo. 
Dentre as complicações tardias da intubação endotraqueal, destaca-se a estenose de traqueia, com ou sem traqueomalácia ${ }^{11-16}$. A estenose traqueal está relacionada principalmente à hiperinsuflação do balonete ("cuff"), acima de 1,5 vezes o diâmetro da traqueia normal. Em casos mais graves, a hiperinsuflação do balonete pode levar até à ruptura traqueal. A TC do tórax é utilizada para detecção da estenose, assim como no planejamento cirúrgico do tratamento. A fase expiratória ou o estudo dinâmico são importantes na detecção da traqueomalácia ${ }^{12}$, quando há o colapso excessivo da traqueia durante a expiração, com redução de calibre ou área superior a $70 \%$ quando comparado à fase inspiratória. Nos casos de traqueostomia, a estenose pode ocorrer também ao nível do traqueostoma.

Há ainda outras complicações relacionadas à ventilação mecânica, principalmente se realizada com pressão positiva ou de maneira prolongada. 0 barotrauma pode levar a pneumotórax, pneumomediastino, enfisema intersticial pulmonar e enfisema de partes moles ${ }^{2}$. A ventilação prolongada aumento o risco de pneumonia aspirativa, pneumonia hospitalar e atelectasias. Em todas estas situações, em geral, o RX de tórax é o primeiro exame de imagem a ser realizado para confirmar a suspeita clínica.

\section{Sondas digestivas}

As sondas digestivas mais comumente são nasogástricas ou nasoentéricas e servem para aspiração de conteúdo, administração de medicamentos e nutrição. A sonda gástrica deve estar com a extremidade no interior do estômago, além da $\operatorname{cardia}^{7,9}$ (abaixo da transição esofagogástrica e do diafragma, no estômago tópico), enquanto a sonda entérica deve estar idealmente na segunda porção do duodeno, além da região pilórica, a direita da linha média. Em geral, as pontas das sondas são radiopacas, para facilitar sua identificação no $\mathrm{RX}$ (figura 4). Antes de verificar o posicionamento correto das sondas digestivas pelo RX, é importante se certificar de que o paciente não possui uma hérnia gástrica hiatal volumosa, mal rotação intestinal ou cirurgia prévia no trato gastrointestinal alto (como gastrectomia parcial). Estas condições podem levar a interpretação errônea do posicionamento das sondas.

A sonda gástrica deve estar com extremidade no interior do estômago, enquando a sonda entérica deve ter extremidade idealmente na segunda porção do duodeno.

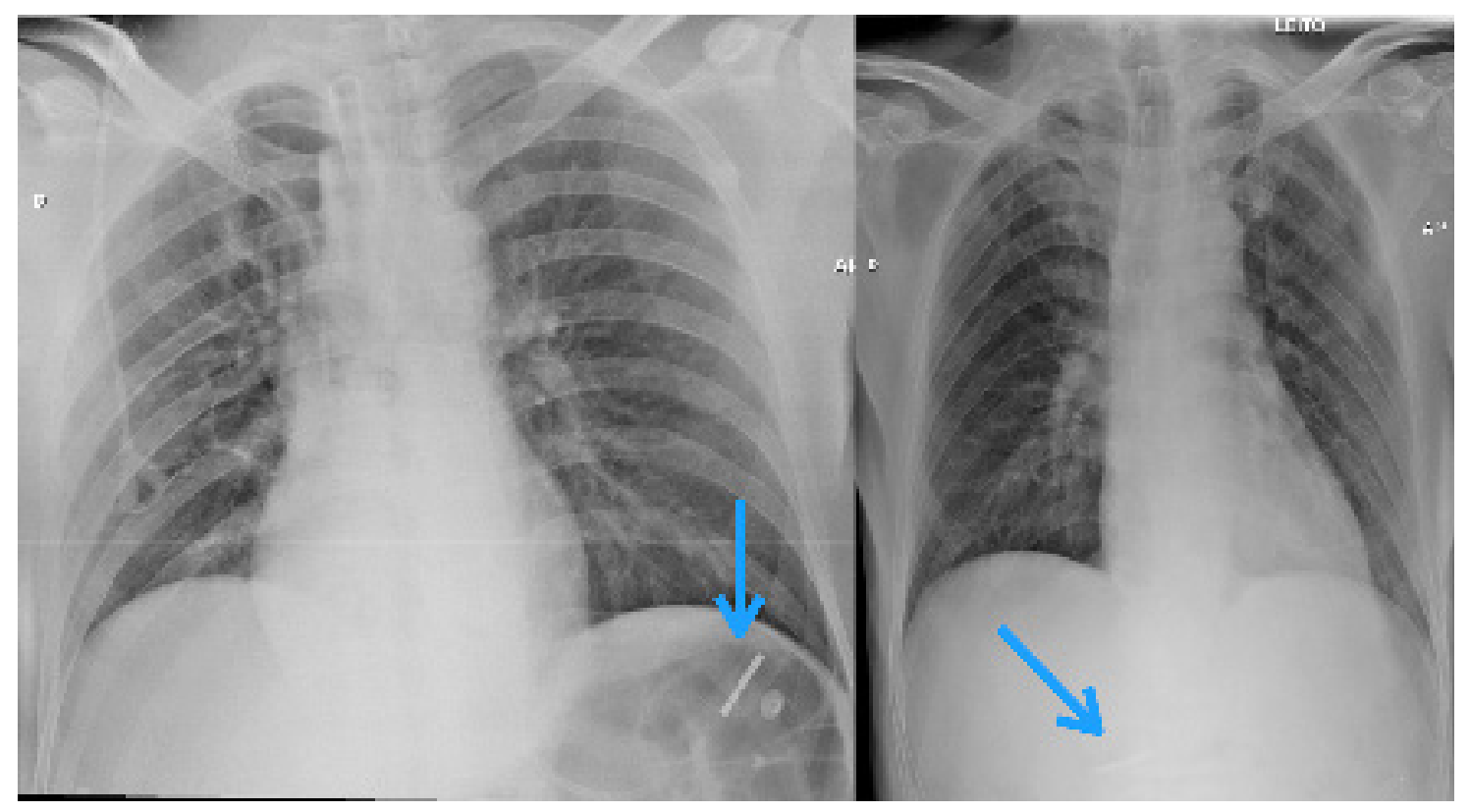

Figura 4: A esquerda, sonda nasogástrica bem posicionada. Há também cateter de acesso venoso central e cânula de traqueostomia, ambos adequadamente posicionados. A direita, sonda nasoentérica com extremidade corretamente posicionada a direita da linha média. 
As complicações mais comuns relacionadas às sondas digestivas incluem o posicionamento errado, com inserção incompleta ou aspecto enrolado da sonda na faringe ou esôfago ${ }^{2,9}$. Por vezes, a sonda pode ser inserida na árvore traqueobrônquica, e menos comumente, mas com pior repercussão clínica, pode haver perfuração e a extremidade ser identificada no pulmão, cavidade pleural e mediastino ${ }^{17}$. Na radiografia, o mal posicionamento pode ser identificado pela posição anômala do trajeto ou da extremidade da sonda (figura 5). As grafias em duas incidências podem ajudar. Ainda, após a administração de medicação ou nutrição, o RX pode mostrar o rápido desenvolvimento de derrame pleural, alargamento mediastinal, pneumotórax ou pneumomediastino, aumentando a suspeição para o mal posicionamento da sonda².

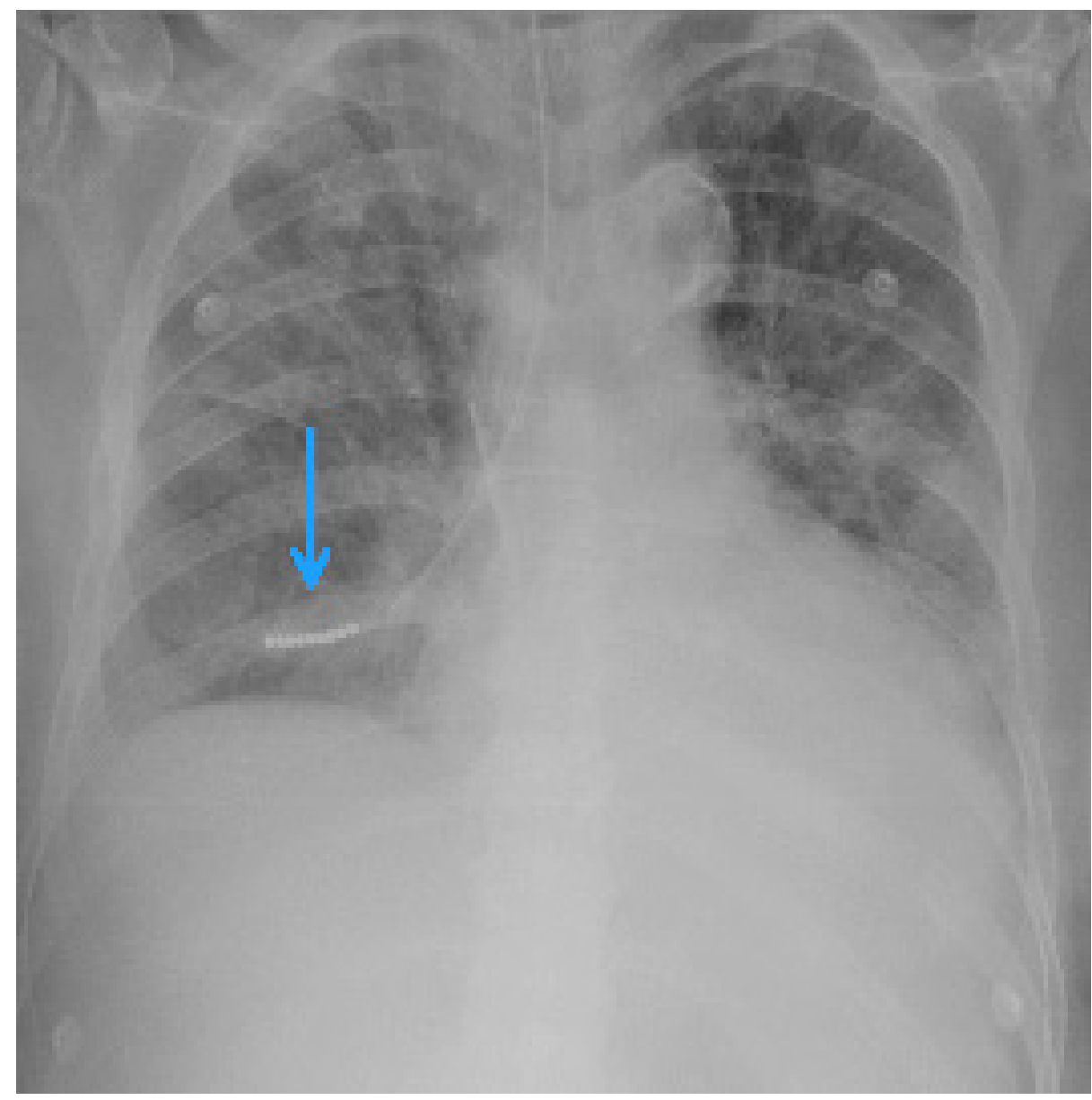

Figura 5: Sonda digestiva mal posicionada, com extremidade em projeção de ramo de brônquio lobar inferior direito. Há também cateter venoso central com extremidade distal na transição entre veia cava superior e átrio direito, e imagens de plugs de monitorização cardíaca.

Complicações da sondagem digestiva incluem também pneumonia aspirativa, abscesso pulmonar, empiema e mediastinite. Nestas situações, a TC de tórax com contraste pode ser importante, para firmar o diagnóstico e auxiliar no planejamento terapêutico².

\section{Dreno torácico}

A drenagem torácica é utilizada para tratamento do derrame pleural e do pneumotórax. Na radiografia, é possível identificar o trajeto, a ponta e os orifícios laterais do dreno. A posição do dreno 
depende do objetivo da drenagem. Para tratamento do pneumotórax, o dreno pode ser mais alto e deve cursar lateral e superiormente com extremidade próximo à região apical. Para tratamento dos derrames pleurais, o dreno pode ser inserido mais baixo e o trajeto posterior e inferior, com extremidade em projeção basal ou lateral (figuras 6 e 7 $)^{2,18}$. Nos casos de derrame pleural loculado ou septado, o dreno deve cursar através da coleção ou coleções. Às vezes, o dreno torácico pode ser substituído por uma valva de Heimlich ou por um cateter pig tail, este mais fino.

A posição do dreno de tórax depende do objetivo. No pneumotórax, é mais alto e cursa superiormente. No derrame, pode ser mais baixo e ter trajeto basal. Nos derrames complexos, deve ter localização específica de acordo com a posição das coleções.

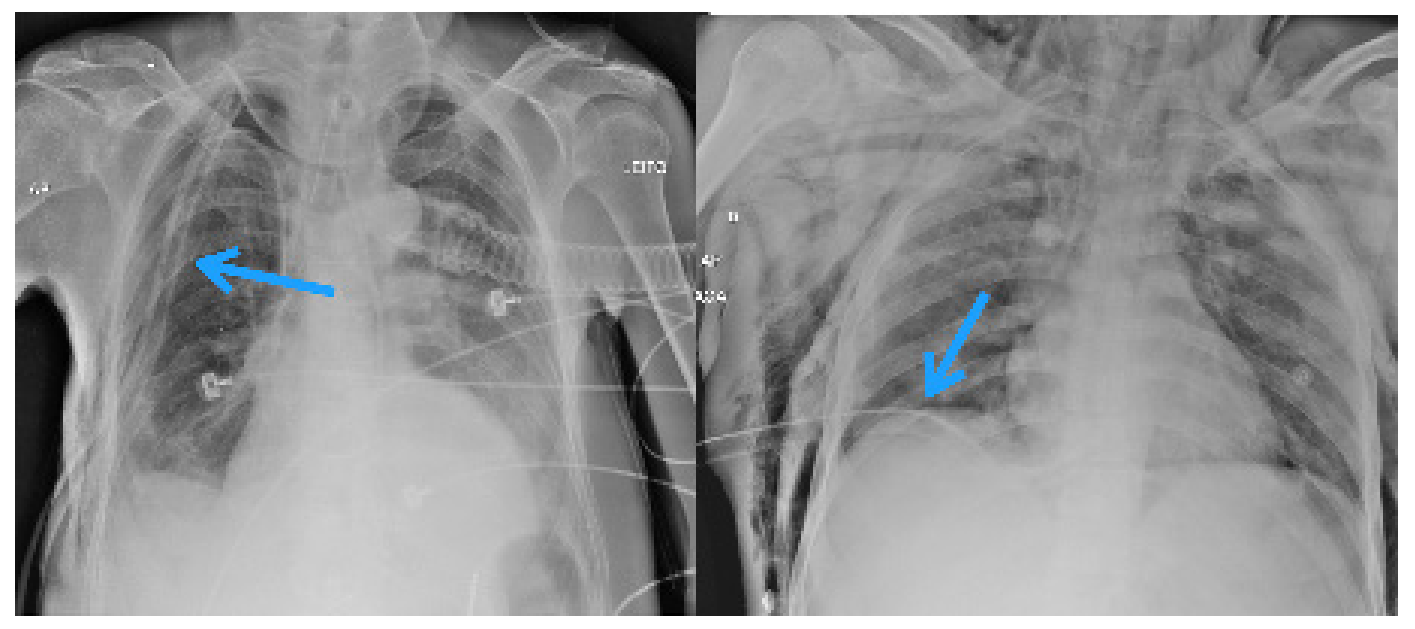

Figura 6: A esquerda, paciente com pneumonia de base esquerda e pneumotórax pós punção venosa a direita, drenado adequadamente. Notar também a cânula de traqueostomia, o cateter venoso central e a sonda nasogástrica, todos adequados. Aparece ainda na grafia o tubo condutor da ventilação mecânica. A direita, drenagem de hemopneumotórax a direita após acidente de moto x carro. Há também importante enfisema de partes moles.

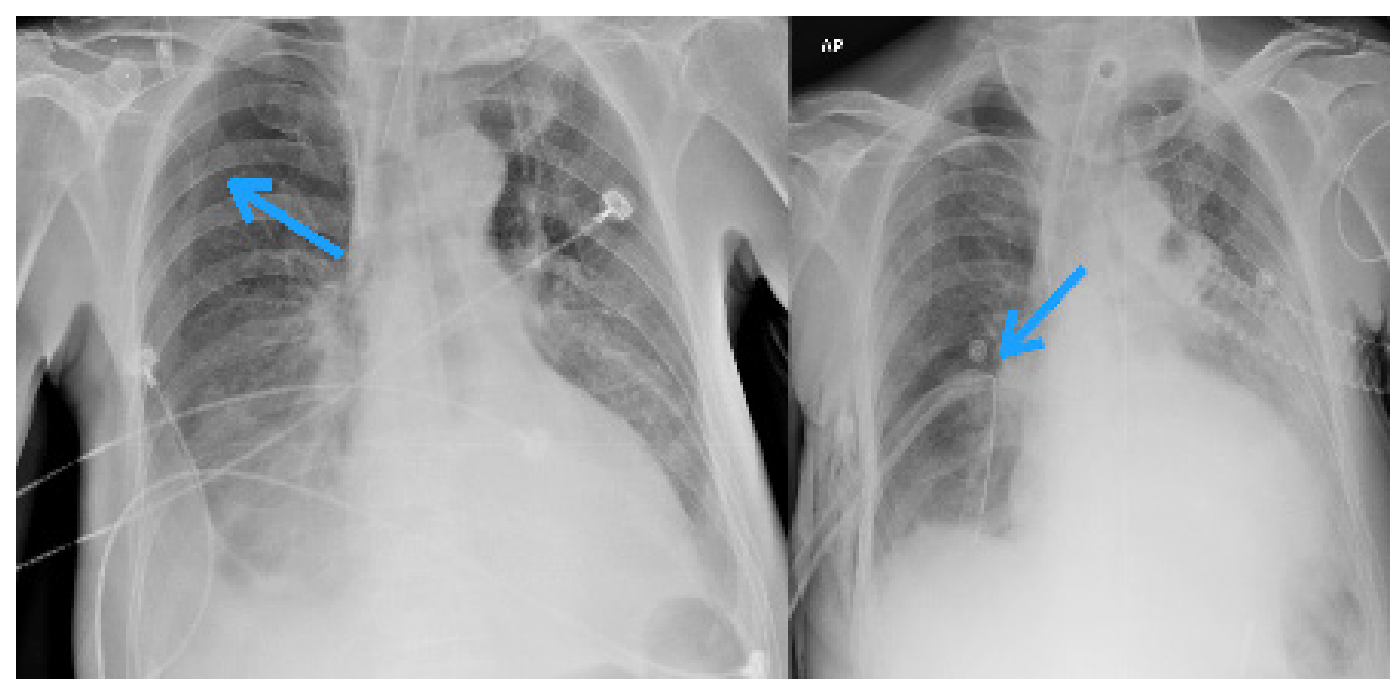

Figura 7: Paciente com pneumonia de base esquerda e pneumotórax pós punção venosa a direita (mesmo paciente da figura 6). Na imagem a direita, o primeiro dreno torácico colocado de maneira incorreta, em trajeto interfissural e dobrado sobre si. Posteriormente, o dreno foi recolocado de maneira correta (figura 6). 
Se o dreno para de drenar ar ou líquido, deve-se suspeitar de mal posicionamento ${ }^{3}$. O orifício proximal do dreno deve estar no interior do tórax, medialmente a borda interna das costelas, caso contrário indica inserção incompleta. O dreno pode também ser erroneamente inserido pelo tecido subcutâneo do paciente, em posição interfissural ou mesmo intrapulmonar (figura 7). A obtenção de grafias em mais de uma incidência ajuda a confirmar a suspeita, além da realização da TC quando necessário. No caso de laceração pulmonar, complicações mais graves podem acontecer, como a formação de hematoma parenquimatoso, infarto pulmonar e fístula broncopleural.

Outras causas de drenagem ineficiente incluem o acotovelamento do dreno ("dobrado sobre si"), entupimento por coágulos ou debris ${ }^{18} \mathrm{e}$ oclusão da ponta por justaposição no mediastino ou parede torácica ${ }^{19}$. A perfuração de órgãos mediastinais, do diafragma ou de órgãos abdominais é complicação rara, mas grave ${ }^{18}$.

\section{Aparatos vasculares}

O exame de RX de tórax deve ser realizado para verificar a posição e sinais de complicações dos seguintes aparatos vasculares:

- cateter venoso central

- cateter arterial pulmonar (Swan-Ganz)

- marcapasso cardíaco

\section{Cateter venoso central}

O cateter de acesso venoso central é utilizado em pacientes graves para administração de medicações endovenosas e monitorização da pressão venosa, sendo posicionados após punção jugular, subclávia ou femoral ${ }^{4}$. Nos pacientes oncológicos em tratamento com quimioterapia, o cateter pode estar acoplado a um reservatório fixo, este, na maioria das vezes, fixado no subcutâneo da parede torácica anterior ("cateter totalmente implantado"). A ponta do cateter venoso deve estar posicionada ao nível da veia cava superior (VCS), logo acima do átrio direito ${ }^{8,10 "}$ e o RX de tórax deve sempre ser feito após uma punção central (ver figuras de 1 a 7). O cateter intra-atrial deve ser evitado, pois, apesar de raro, há o risco de arritmia, ruptura miocárdica e tamponamento cardíaco ${ }^{9}$.

A complicação mais comum é o posicionamento inadequado do cateter, por vezes associado a torção ou acotovelamento ${ }^{10}$ (figura 8 ). Se necessário, outras incidências podem auxiliar a identificar que o cateter não se encontra em projeção da VCS, podendo estar na veia cava inferior, veia ázigos ou ventrículo direito. Variantes anatômicas, como persistência da veia cava superior esquerda, drenagem venosa pulmonar anômala e defeitos dos septos cardíacos podem também levar a trajeto anômalo do cateter e gerar confusão ${ }^{4}$.

A ponta do cateter venoso central deve estar na projeção da veia cava superior no exame de RX, abaixo da primeira costela anterior e logo acima do contorno do átrio direito.

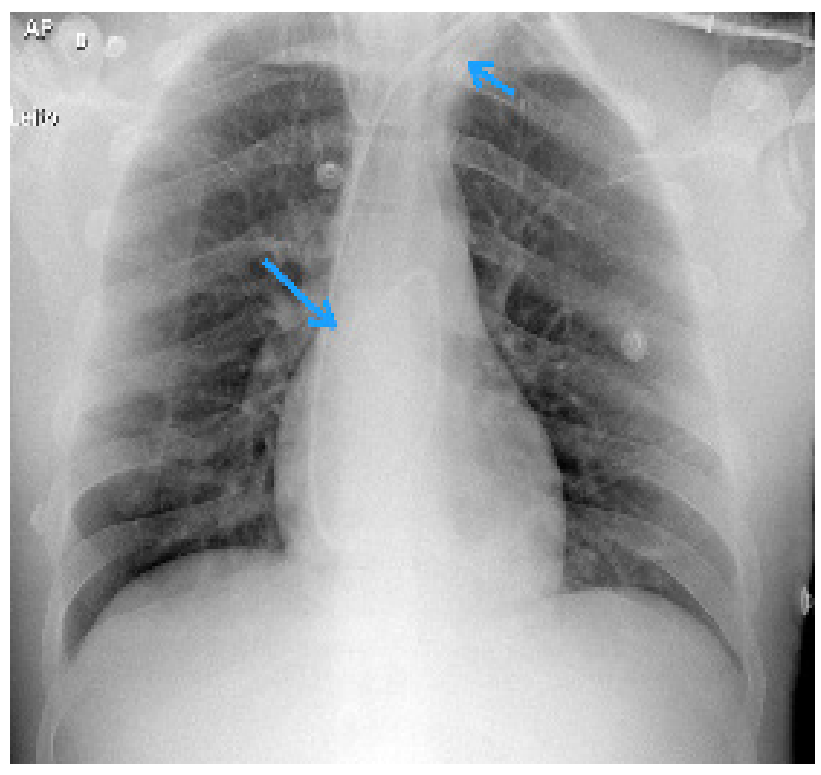

Figura 8: Cateter de Swan-Ganz adequadamente posicionado em região do hilo pulmonar direito. Notar ainda cateter venoso central inserido via jugular interna direita que cursou erroneamente para a veia subclávia esquerda.

Outras complicações do acesso venoso central incluem o pneumotórax, hemotórax, hematoma extrapleural e hematoma mediastinal ${ }^{10}$ (figuras 6 e 7 ). Todas estas condições podem ser identificadas no 
RX de tórax e, quando necessário, pode-se complementar a avaliação com grafias após injeção de pequena quantidade de meio de contraste iodado pelo cateter ou com a TC. Complicações menos comuns e de diagnóstico mais difícil incluem a trombose completa ou parcial, ruptura, fragmentação e migração de fragmento do cateter (incluindo a embolia pulmonar não trombótica $)^{4}$.

\section{Cateter arterial pulmonar}

O cateter de Swan-Ganz é utilizado para monitorização hemodinâmica circulatória, ajudando inclusive na diferenciação entre edema pulmonar cardiogênico e não cardiogênico. É inserido por via venosa e a ponta deve estar idealmente na artéria pulmonar direita ou esquerda, não se devendo estender aos ramos interlobares. Desta maneira, no RX de tórax, a ponta não deve estar além de $2 \mathrm{~cm}$ de distância do hilo pulmonar ${ }^{10}$ (figura 8).

No RX de tórax, a ponta do cateter de Swan-Ganz deve estar na região do hilo pulmonar direito ou esquerdo.
Além das complicações semelhantes às observadas em casos de acesso venoso central, o cateter de Swan-Ganz pode levar a infarto pulmonar e, mais raramente, a dissecção da artéria pulmonar ${ }^{6}$.

\section{Marcapasso cardíaco}

O marcapasso cardíaco pode ser temporário ou definitivo e pode ser utilizado para tratamento de diferentes distúrbios de condução no coração. O marcapasso temporário pode ser inserido por via venosa, transtorácica, epicárdica ou transesofágica. Os marcapassos definitivos possuem um dispositivo gerador de pulso que fica implantado no subcutâneo anterior do tórax e cabos com eletrodos cardíacos, estes podendo ser único ou múltiplos. A apresentação mais comum é de dois cabos finos e uniformes, um com extremidade em átrio direito e outro no ventrículo direito (figura 9). Em casos de marcapasso biventricular ou terapia de ressincronização, há um terceiro $\mathrm{cabo}^{3}$, que mais comumente cursa em seio coronariano até uma veia cardíaca esquerda para estimular o ventrículo esquerdo, ou pode ter posição epicárdica esquerda.

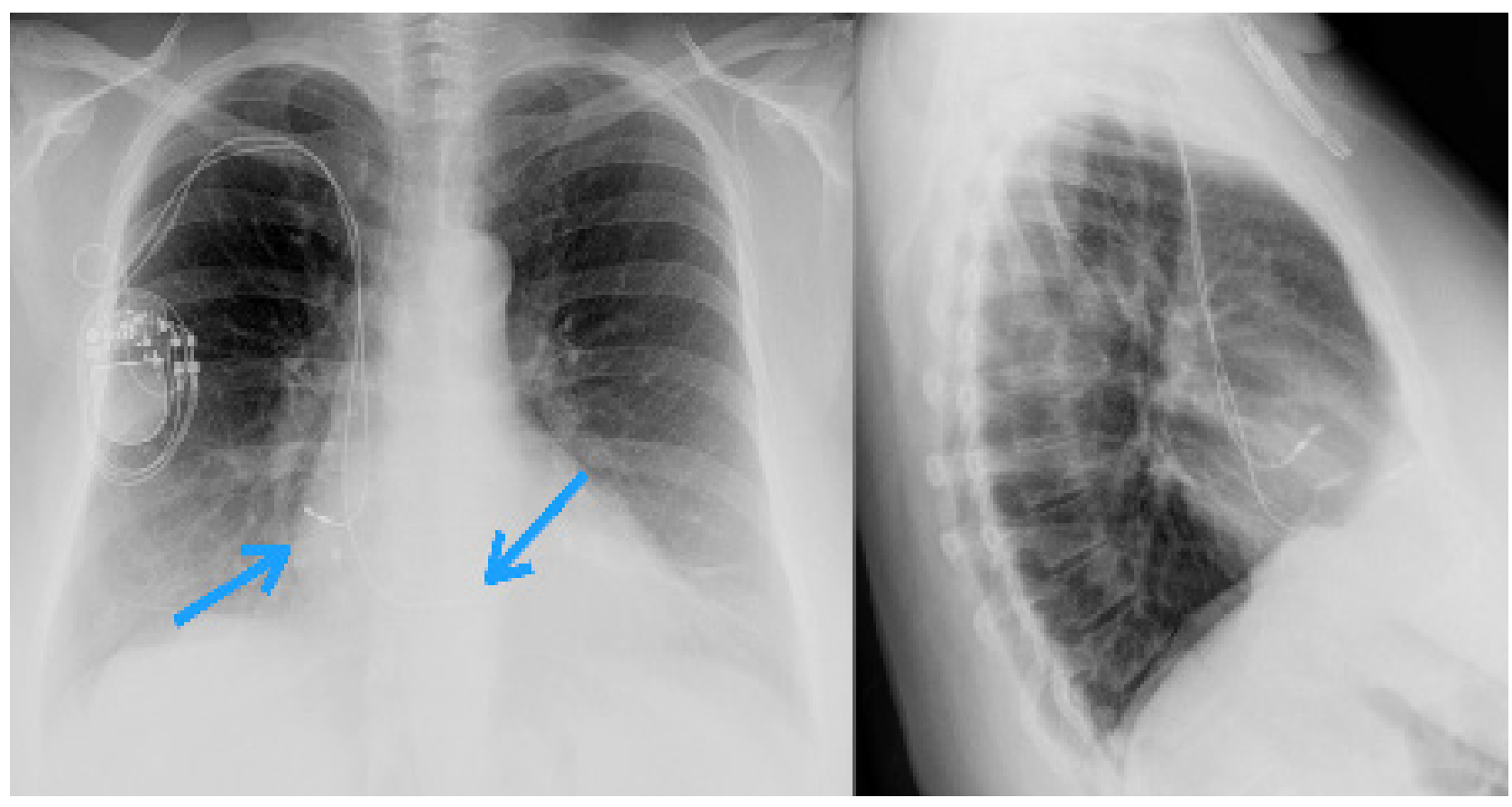

Figura 9: Aparelho de marcapasso cardíaco com dois eletrodos íntegros com extremidades em 
Nos pacientes com risco de taquicardia ou fibrilação ventricular, o dispositivo de cardioversão-desfibrilação (CDI) pode estar associado ao mecanismo de marcapasso ${ }^{20,21}$ (figura 10). O CDI em geral tem gerador maior, possui cabo heterogêneo com "mola" na região distal e extremidade no ventrículo direito, e quando da presença de dois cabos, o segundo em VCS ou veiabraquicefálica.

O marcapasso cardíaco definitivo mais comumente possui dois cabos finos de eletrodos, um em átrio e outro em ventrículo direito.
Às vezes, há um terceiro cabo relacionado ao ventrículo esquerdo (venoso ou epicárdico). O CDI mais comumente tem um cabo com "mola distal" e extremidade em ventrículo direito.

As mesmas complicações relacionadas aos cateteres venosos podem ocorrer durante a passagem do marcapasso cardíaco. Adicionalmente, pode ocorrer perfuração do miocárdio, identificado no RX quando a ponta do cabo se estende além da borda cardíaca ${ }^{10}$ É preciso estar atento também para o deslocamento e as fraturas dos cabos, em geral fáceis de serem identificados no $\mathrm{RX}^{4,22}$ (figura 10).

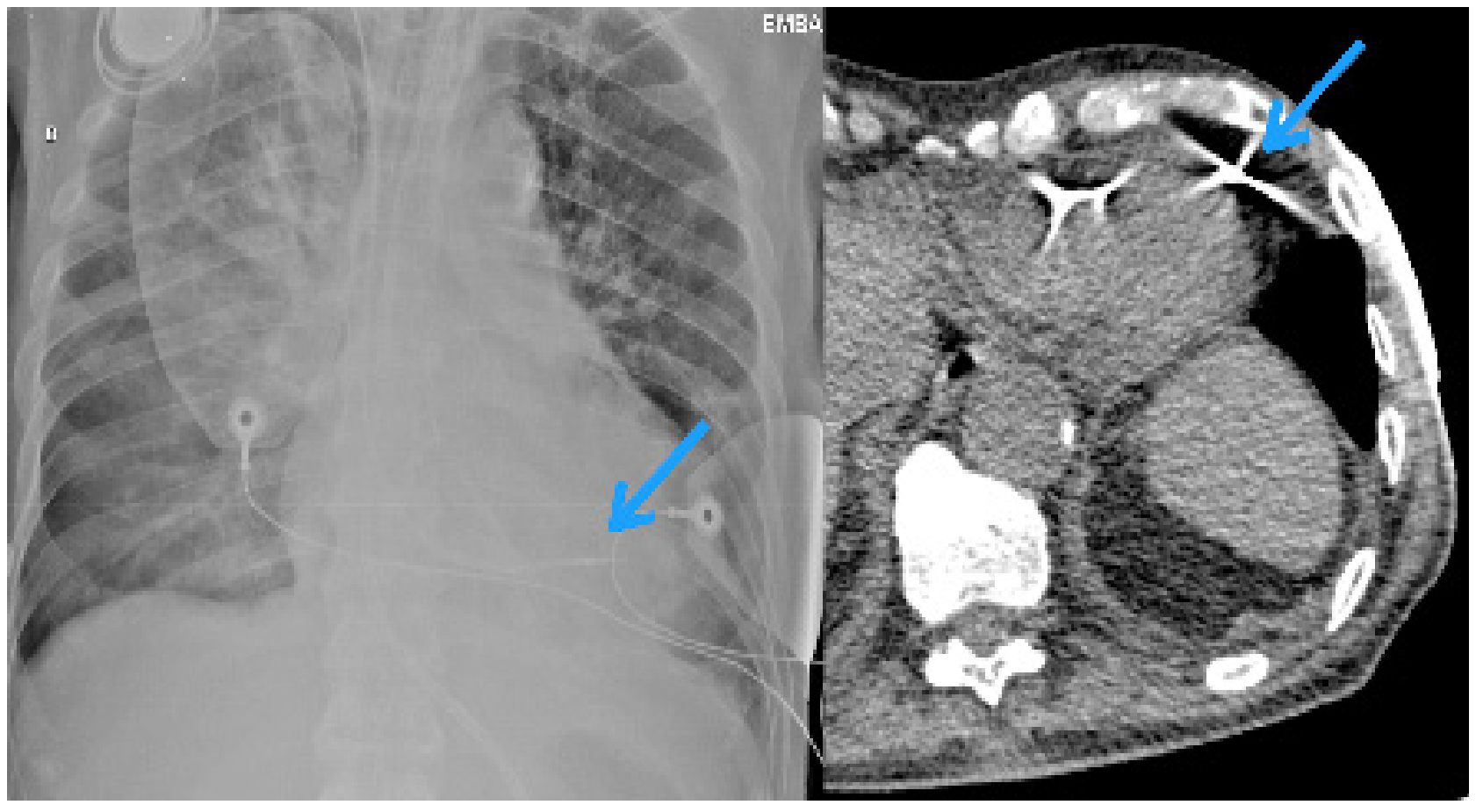

Figura 10: Controle pós instalação de CDI em paciente com arritmia cardíaca (RX a esquerda). O aparelho não funcionava corretamente e a TC (a direita) mostrou que a extremidade do cabo havia perfurado o miocárdio do ventrículo direito e estava no pericárdio. Notar no RX a presença de sonda digestiva (ponta não identificada) e os eletrodos de monitorização.

\section{Outros aparatos médicos}

Outros aparatos médicos comumente identificados ao RX de tórax incluem:

- $\quad$ plugs e fios de monitorização cardíaca

- $\quad$ tubos de ventiladores mecânicos

- $\quad$ sondas sobrepostas ao corpo do paciente
- $\quad$ cateter de derivação ventriculoperitoneal

- fios de esternorrafia e clips mediastinais

Há muitos outros dispositivos médicos que comumente são identificados no exame de RX de tórax, principalmente de pacientes graves, internados ou em avaliação de urgência / emergência. Mesmo que o RX não seja utilizado para verificar a posição ou complicações relacionadas a estes 
aparatos, é importante saber reconhecer seu aspecto radiográfico para não confundir com alterações patológicas ou imagens de corpo estranho ${ }^{3}$. Muitas vezes, no exame em AP do paciente grave, não é possível retirar os equipamentos médicos de monitorização e suporte do paciente para a realização da radiografia ${ }^{3}$. Assim, é preciso saber reconhecer no RX os plugs e fios de monitorização cardíaca, tubos e conexões dos aparelhos de ventilação mecânica, sondas e cateteres sobrepostos ao corpo do paciente, para não confundir com dispositivos no interior do organismo ou outro tipo de lesão torácica (ver figuras de 1 a 10).
Outro aparato que pode ser identificado no RX de tórax é o cateter de derivação ventriculoperitoneal, utilizado no tratamento de hidrocefalia não obstrutiva ${ }^{23}$ (figura 11). A porção torácica do cateter cursa no tecido subcutâneo longitudinalmente, comunicando a porção cefálica (esta geralmente ligada a uma valva) com a porção abdominal. Há complicações que podem ser identificadas no RX de tórax, principalmente a ruptura do cateter e o surgimento de derrame pleural, mas estas são incomuns.

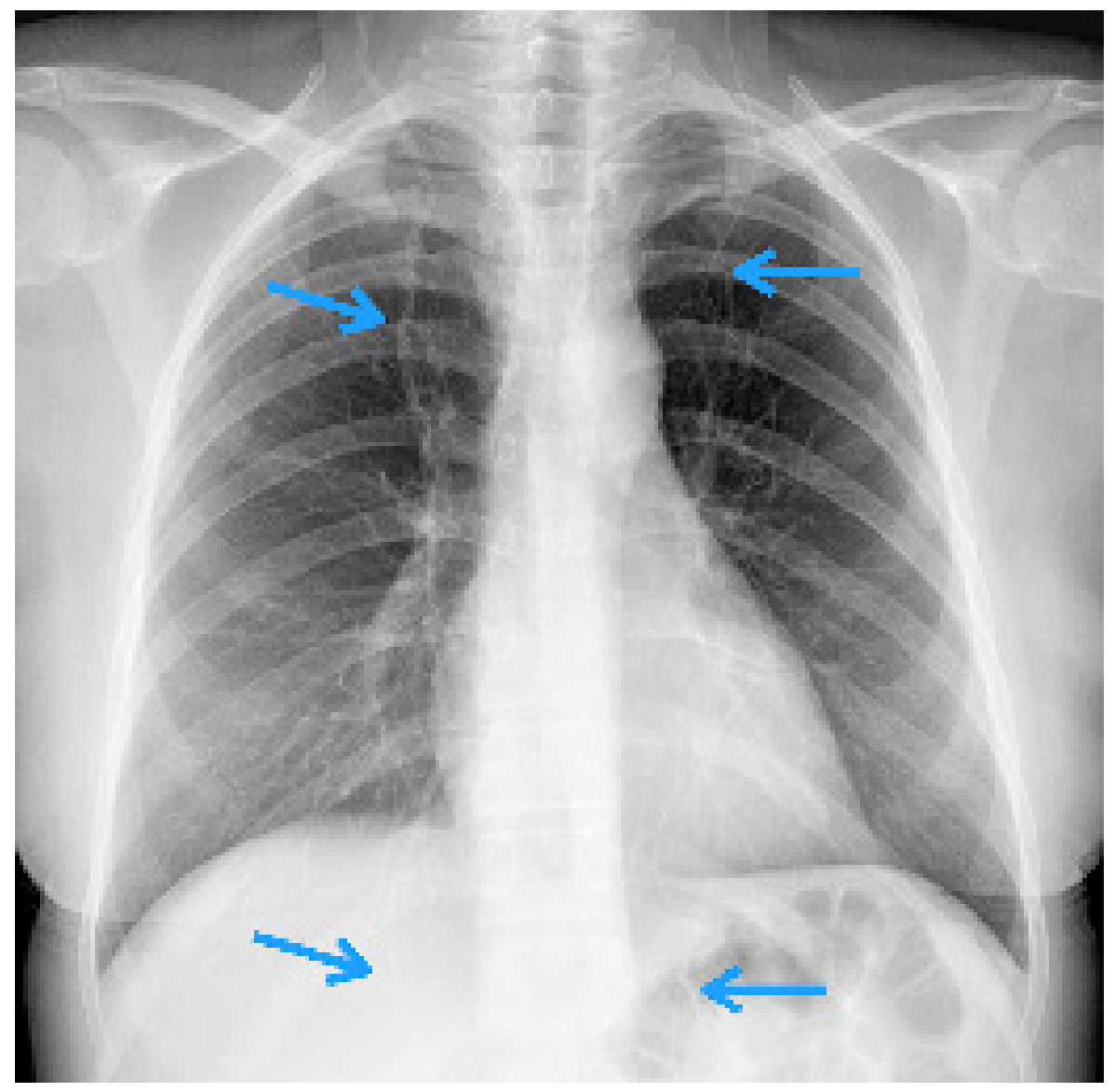

Figura 11: Paciente com dois cateteres de derivação ventriculoperitoneal cursando anteriormente na parede torácica, um a direita e outro, mais fino, a esquerda.

É importante também saber identificar os aparatos cirúrgicos no tórax, principalmente os relacionados à cirurgia cardíaca, torácica e os materiais ortopédicos da coluna, para não confundir com imagens de corpo estranho ${ }^{3,23}$ (figura 12).
Clips metálicos mediastinais são pequenos, mas visíveis, e em geral estão relacionados a cirurgia de revascularização miocárdica ou ressecção de tumores mediastinais ${ }^{3}$. Os fios metálicos de esternorrafia são facilmente identificados no RX e, 
por vezes, a fratura de um dos fios pode ser identificada ao exame. Outras complicações, como infecção e deficiência de consolidação óssea, requerem outros exames com a TC ou ressonância magnética $(\mathrm{RM})^{24,25}$. Próteses valvares cardíacas, stents coronarianos e de ramos do arco aórtico, próteses endovasculares da aorta (reparo de aneurisma ou tratamento de dissecção), stents traqueais, brônquicos ou esofágicos podem também ser identificados no RX de tórax ${ }^{3}$. Para mensuração do $\mathrm{pH}$ esofágico, usa-se uma sonda de pHmetria com ponta radiopaca, sendo que o RX é feito para identificar o posicionamento adequado da extremidade da sonda no esôfago distal ${ }^{3}$.

Obviamente que, se ao exame de RX do tórax, for identificada imagem de outro tipo de aparato cirúrgico, como compresssa, gaze, pinça, agulha ou afastador, deve ser averiguado a possibilidade de iatrogenia, em que tal aparato foi esquecido du- rante um procedimento cirúrgico, caracterizando um corpo estranho intratorácico ${ }^{26}$. Atualmente, até as compressas cirúrgicas possuem fios radiopacos de marcação, para auxiliar em sua identificação. Nos casos crônicos, em que um instrumento médico é esquecido no interior do corpo do paciente, pode-se formar uma reação inflamatória granulomatosa grave, gerando aspecto de massa, que pode ser até confundida com neoplasia (figura 12). Estas massas, quando relacionadas a compressas ou gazes, são chamadas de gossipibomas ${ }^{27}$.

A imagem de corpo estranho deve ser suspeitada quando se identifica no RX um dispositivo ou equipamento médico não esperado, por vezes, relacionado a massa ou coleção. Por isso, é importante saber reconhecer todos os aparatos médicos e seu aspecto habitual na imagem radiográfica.

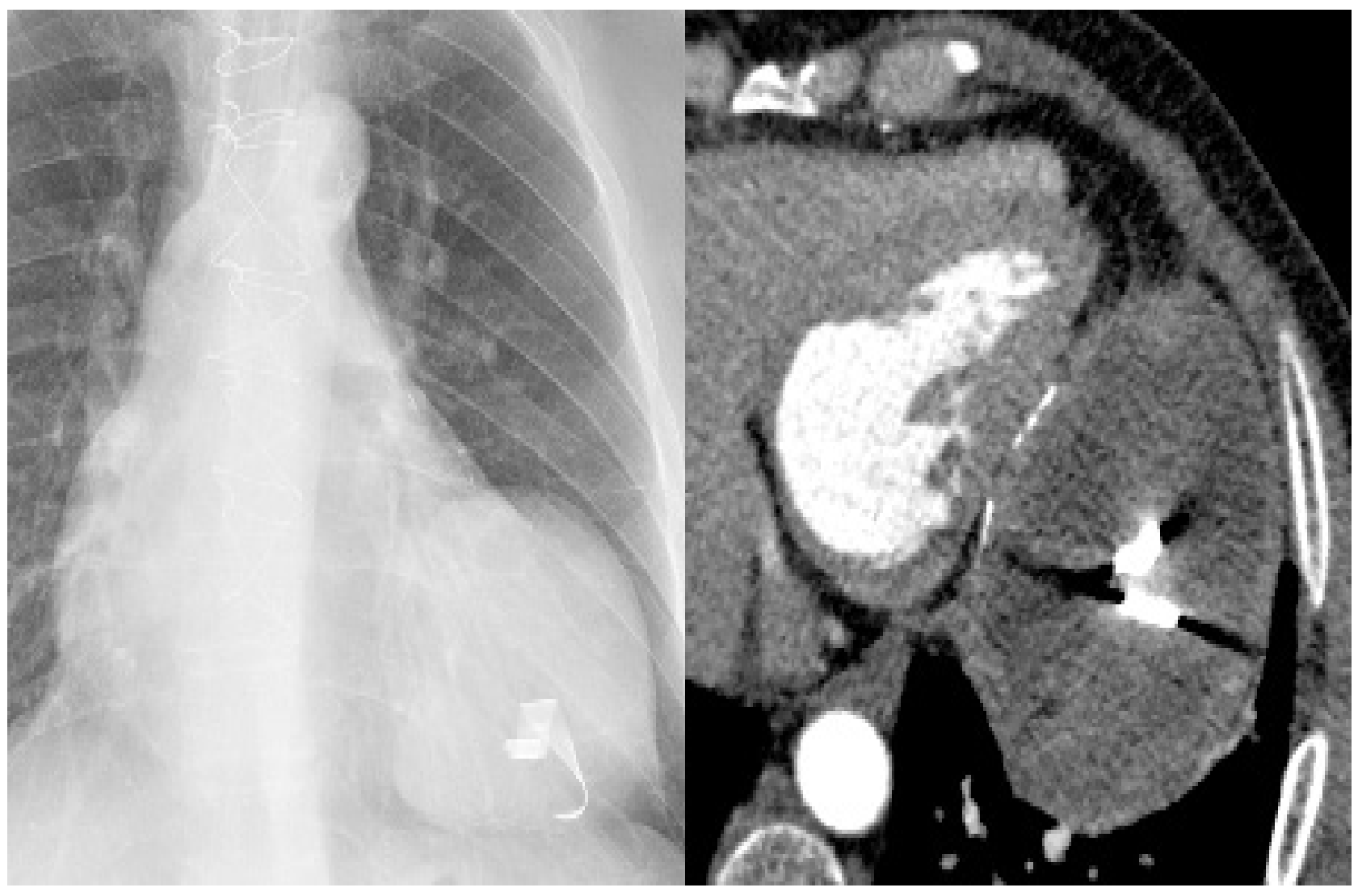

Figura 12: Controle radiográfico tardio pós-operatório de cirurgia de revascularização miocárdica mostrou imagem com aspecto de massa junto ao coração associado a imagem metálica no interior, lembrando um afastado cirúrgico. A esquerda, no $\mathrm{RX}$, é possível ver os fios metálicos de esternorrafia e os clips cirúrgicos. A direita, a imagem de TC mostra tratar-se de uma grande massa de provável granuloma de corpo estranho em região epicárdica. 


\section{CONCLUSÃO}

Neste texto foram apresentados e discutidos os seguintes temas:

- O aspecto radiográfico dos principais aparatos médicos utilizados no tórax, como tubos, sondas e cateteres

- o posicionamento adequado de vários dispositivos, vasculares e não vasculares

- $\quad$ as principais complicações relacionadas à instalação e permanência de alguns aparatos, identificáveis ao RX de tórax

\section{REFERÊNCIAS}

1. Suh RD, GenshaftSJ, Kirsch J, Kanne JP, Chung JH, Donnelly $E F$, et al. ACR Appropriateness Criteria ${ }^{\circledR}$ Intensive Care Unit Patients. J Thorac Imaging. 2015;30(6):W63-W65. doi:10.1097/RTI.0000000000000174

2. Godoy MCB, Leitman BS, Groot PM, Vlahos I, Naidich DP. Chest Radiography in the ICU: Part 1, Evaluation of Airway, Enteric, and Pleural Tubes. Am J Roentgenol. 2012;198(3):563-571. doi:10.2214/AJR.10.7226

3. Hunter TB, Taljanovic MS, Tsau PH, Berger WG, Standen JR. Medical Devices of the Chest. Radiographics. 2004;24(6):1725-1746. doi:10.1148/rg.246045031

4. Godoy MCB, Leitman BS, Groot PM, Vlahos I, Naidich DP. Chest Radiography in the ICU: Part 2, Evaluation of Cardiovascular Lines and Other Devices. Am J Roentgenol. 2012;198(3):572-581. doi:10.2214/AJR.11.8124

5. Lacey G De, Morley S, Berman L. Radiografia Do Tórax Um Guia Prático. Elsevier Editora Ltda; 2010.

6. Wiener MD, Garay SM, Leitman BS, Wiener DN, Ravin CE. Imaging of the intensive care unit patient. Clin Chest Med. 1991;12(1):169-198. http://www.ncbi.nlm.nih. gov/pubmed/1901259.

7. Hill JR, Horner PE, Primack SL. ICU Imaging. Clin Chest Med. 2008;29(1):59-76. doi:10.1016/j. ccm.2007.11.005

8. Rubinowitz AN, Siegel MD, Tocino I. Thoracic Imaging in the ICU. Crit Care Clin. 2007;23(3):539-573. doi:10.1016/j.ccc.2007.06.001

9. Wechsler RJ, Steiner RM, Kinori I. Monitoring the monitors: The radiology of thoracic catheters, wires, and tubes. Semin Roentgenol. 1988;23(1):61-84. doi:10.1016/S0037-198X(88)80018-X

10. MCLoud TC. Thoracic Radiology: The Requisites. In: Elsevier; 2010.

11. Wain JC. Postintubation Tracheal Stenosis. Semin Thorac Cardiovasc Surg. 2009;21(3):284-289. doi:10.1053/j. semtcvs.2009.08.001
12. Heidinger $B H$, Occhipinti $M$, Eisenberg $R L$, Bankier $A A$. Imaging of Large Airways Disorders. Am J Roentgenol. 2015;205(1):41-56. doi:10.2214/AJR.14.13857

13. Boiselle PM. Imaging of the Large Airways. Clin Chest Med. 2008;29(1):181-193. doi:10.1016/j.ccm.2007.11.002

14. Carden KA, Boiselle PM, Waltz DA, Ernst A. Tracheomalacia and Tracheobronchomalacia in Children and Adults. Chest. 2005;127(3):984-1005. doi:10.1378/chest.127.3.984

15. Ridge CA, O'Donnell CR, Lee EY, Majid A, Boiselle PM. Tracheobronchomalacia: current concepts and controversies. J Thorac Imaging. 2011;26(4):278-289. doi:10.1097/RTI.0b013e3182203342

16. Boiselle PM, Ernst A. Tracheal Morphology in Patients With Tracheomalacia: Prevalence of Inspiratory Lunate and Expiratory "Frown" Shapes. J Thorac Imaging. 2006;21(3):190196. doi: 10.1097/01.rti.0000213647.42041.d0

17. Bankier AA, Wiesmayr MN, Henk C, Turetschek K, WinkeIbauer F, Mallek $R$, et al. Radiographic detection of intrabronchial malpositions of nasogastric tubes and subsequent complications in intensive care unit patients. Intensive Care Med. 1997;23(4):406-410. doi:10.1007/s001340050348

18. Dev SP, Nascimiento B, Simone C, Chien V. Chest-Tube Insertion. N Engl J Med. 2007;357(15):e15. doi: 10.1056/NEJMvcm071974

19. Hunter TB. Tubes, lines, catheters, and other interesting devices. Curr Probl Diagn Radiol. 1995;24(2):55-108. doi:10.1016/S0363-0188(95)80002-6

20. DiMarco JP. Implantable Cardioverter-Defibrillators. N Engl J Med. 2003;349(19):1836-1847. doi:10.1056/NEJMra035432

21. Drucker EA, Brooks R, Garan H, Sweeney MO, Ruskin JM, McGovern BA, et al. Malfunction of implantable cardioverter defibrillators placed by a nonthoracotomy approach: frequency of malfunction and value of chest radiography in determining cause. Am J Roentgenol. 1995;165(2):275-279. doi:10.2214/ajr.165.2.7618539

22. Chang S-H, Tan C-K, Lee S-H. Fracture of a pacemaker lead. Can Med Assoc J. 2009;181(11):823-823. doi: $10.1503 / \mathrm{cmaj} .082092$

23. Hunter TB, Yoshino MT, Dzioba RB, Light RA, Berger WG. Medical Devices of the Head, Neck, and Spine. Radiographics. 2004;24(1):257-285. doi:10.1148/rg.241035185

24. Li AE, Fishman EK. Evaluation of Complications After Sternotomy Using Single- and Multidetector CT with Three-Dimensional Volume Rendering. Am J Roentgenol. 2003;181(4):1065-1070. doi:10.2214/ajr.181.4.1811065

25. Restrepo CS, Martinez S, Lemos DF, Washington L, McAdams HP, Vargas $D$, et al. Imaging Appearances of the Sternum and Sternoclavicular Joints. Radiographics. 2009;29(3):839-859. doi:10.1148/rg.293055136

26. Karabulut N, Herek D, Kiroglu Y. CT features of intrathoracic gossypiboma (textiloma). Diagnostic Interv Radiol. 2011;17(2):122-4. doi:10.4261/1305-3825.DIR.3120-09.0

27. Madan R, Trotman-Dickenson $B$, Hunsaker AR. Intrathoracic Gossypiboma. Am J Roentgenol. 2007;189(2):W90-W91. doi:10.2214/AJR.07.2250 


\section{MATERIAL SUPLEMENTAR}

\section{EXERCÍCIOS}

1. Em relação ao posicionamento do tubo endotraqueal, assinale a alternativa incorreta.

a) O RX deve ser realizado logo após a intubação

b) A cabeça do paciente deve estar em posição neutra

c) A extremidade deve estar a aprox. $5 \mathrm{~cm}$ da carina

d) Intubação seletiva mais comumente ocorre a esquerda

e) A estenose traqueal é complicação tardia

2. Quais as diferenças entre a sonda nasogástrica e nasoentérica?

3. Qual aparato médico é identificado neste exame? Inserido por que via? O posicionamento está correto? Por quê?

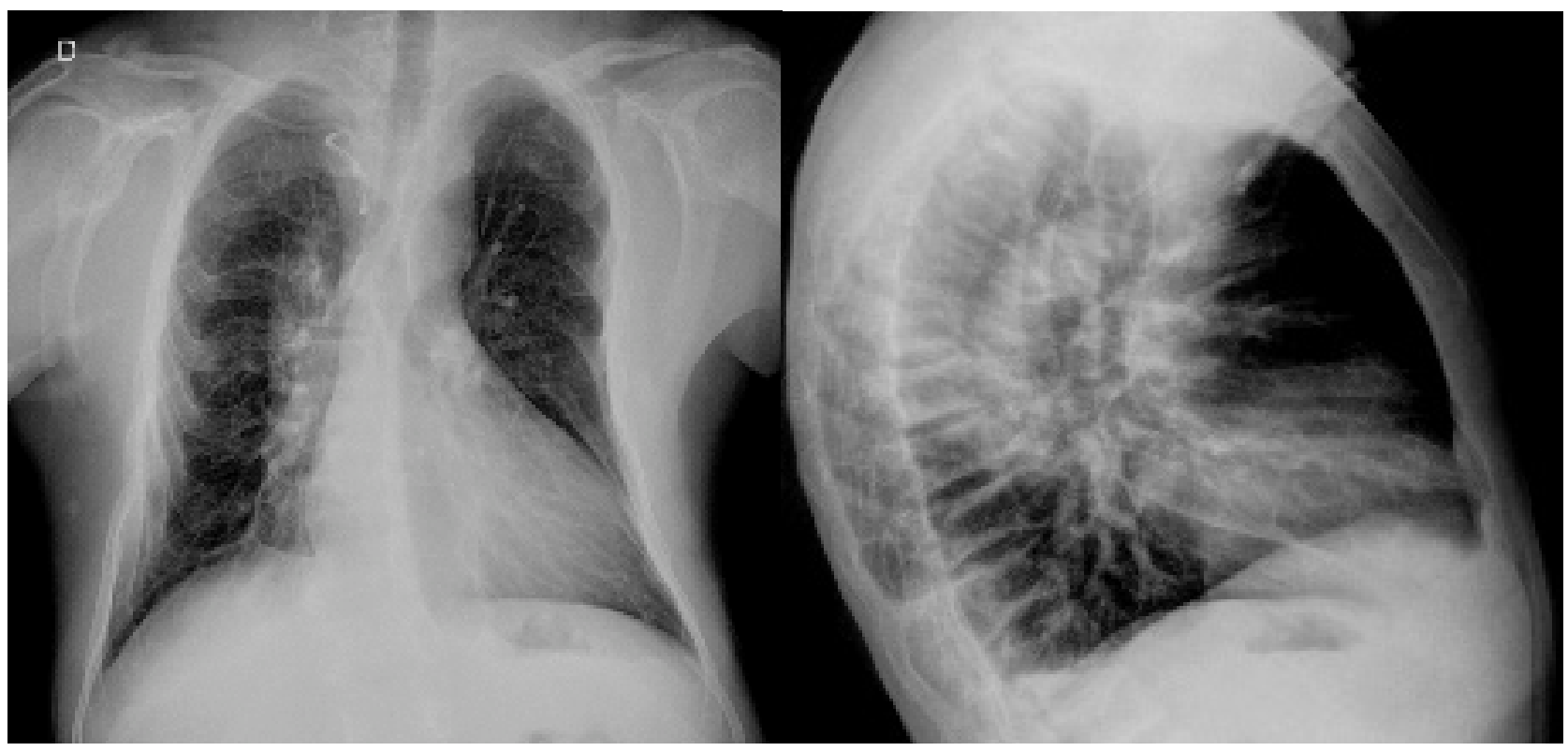


4. Em relação aos dispositivos médicos cardiovasculares, correlacione:
A. Marcapasso cardíaco
B. CDI
C. Cateter de Swan-Ganz
D. Balão intra-aórtico
E. Fios de monitorização cardíaca

( ) Não confundir com sondas ou cateteres

( ) Dois eletrodos, em átrio e ventrículo direito

( ) Um cabo em ventrículo direito

( ) Ponta radiopaca ao nível do botão aórtico

( ) Extremidade na região do hilo pulmonar

5. Cite três posições inadequadas do dreno torácico. 